\title{
Image Processing and Classification, A Method for Plant Disease Detecion
}

\author{
Simranjeet kaur, Geetanjali Babbar, Gagandeep
}

\begin{abstract}
The plant disease detection is the major issue of the computer vision and machine learning. The plant disease detection has the various phases like pre-processing, segmentation, feature extraction and classification. In the existing technique support vector machine is used for the classification. The support vector machine approach has the low accuracy for the plant disease detection and also it can classify data into two classes which affect its performance. The proposed methodology is based on the region based segmentation, textual feature analysis and k-nearest neighbor method is applied for the classification. The proposed method is implemented in MATLAB and results are analyzed in terms of accuracy. The proposed technique has high accuracy and compared to existing technique.
\end{abstract}

\section{Index Terms: Plant disease detection, GLCM, K-mean, KNN}

\section{INTRODUCTION}

Image processing can be described as a type of signal processing. In image processing, the input is applied in form of an image like a picture or video format. The output of image processing will be either an image or a suite of features or metrics in relevance of the given image. This approach is used to extract some valuable information from the raw images obtained from different places. Many methods of image-processing treat the image as 2-dimensional signal and apply standard signal-processing techniques to it. The two situations which go with image processing are identified as The MatLab and MathCAD [11]. Image enhancement, image restoration, image compression, image segmentation, image recognizing, image smoothing are some important techniques of image processing. The techniques of image processing can be used to detect plant diseases. The signs of disease are observed on the leaves, stem and fruit in several cases. The plant leaf showing disease symptoms is considered for the recognition of disease. In this work, some image processing techniques are utilized to detect plant disease. In this work, some fundamental steps to detect and classify plant disease are implemented with the help of image processing. The initial step in image processing is image acquisition. A camera is used to obtain the pictures of the plant leaf. The captured images are in RGB (Red, Green and Blue) format. In this step, a color conversion formation for the RGB leaf picture is formed. Further, a device-independent color space conversion

Revised Manuscript Received on June 15, 2019.

Simranjeet kaur, pursuing MTech from Chandigarh Group Of College ,Landran ,Mohali, India.

Geetanjali Babbar, Assistant Professor in Chandigarh Engineering College, Landran, India.

Dr. Gagandeep, Department of Computer s Science and Engineering, Chandigarh Engineering College (CEC), Landran, Mohali, India. for the color conversion formation is implemented. Various pre-processing techniques are applied for eliminating the noise. After this, Image clipping is performed [7]. In this process, the leaf images are cropped to obtain the required section of the image. In addition, smoothing filter is used to obtain smooth image. The next process is Image enhancement. This process improves the contrast. After image enhancement, the histogram equalization method is applied on the image to make improvements in the plant infection images. This method distributes the intensities of the images. In order to allocate intensity values, the cumulative distribution function is utilized. The next step in image processing is image segmentation. In this process, the image is partitioned into different fractions of identical features or containing some resemblance [8]. Several methods such as otsu' method, k- means clustering and transforming RGB image into HIS model etc are used to perform segmentation. Segmentation can also be performed using Boundary and spot detection algorithm. In this approach, the RGB image is transformed into the HIS model for segmentation process. This approach is used to discover the diseased section of the leaf. In ostu threshold algorithm, the binary images are developed from grey-level images using thresholding. In this method, all the pixels below some threshold are set to zero while all pixels above that threshold are set to one. After image segmentation, feature extraction process is applied. In order to identify an object, the feature extraction technique is very important. The feature extraction process is used in most of the applications of image processing [12]. In order to detect plant disease, some features such as color, texture, morphology, edges etc. are utilized. In this process, some features of the image are extracted on the basis of segmented information and predefined dataset. This extraction may be in the form of statistical, structural, fractal or signal processing. Some methods utilized for feature extraction are color co-occurrence method, Grey Level Co- occurrence Matrices (GLCM), Spatial Gray-level Dependence Matrices (SGDM) method, Gabor Filters, Wavelets Transform and Principal component analysis. The next step after feature extraction is image classification. In this process, various classifiers are used for the classification of images. The Classifiers can be defined in terms of software schedule written on the platform for describing definite features to classify the images. Some linear and non linear classification models for image classification are $\mathrm{K}$ nearest neighbor, Radial basis function, Probabilistic Neural Network (PNN), Convolution neural network, Support vector machine and Back propagation network. These classifiers can be trained and 
tested using MATLAB. A lot of color representations are used by the SVM technique during its implementation [4]. An MLP neural network separates leaves and background. After this, the colors gifts on the leaves are clustered by the suggestion of an unsupervised and undisciplined self-organizing map. The quantity of clusters to be implemented in each case is determined by a genetic algorithm. Subsequently, a Support Vector Machine (SVM) classifier splits infectious and healthy regions. The K-means clustering algorithm classifies objects on the bsis of a set of features into $\mathrm{K}$ number of classes. The objects are classified by minimizing the sum of the squares of the distance among the object and the equivalent cluster. The back propagation neural network (BPNN) is also used for image classification. This classifier builds relationship among recognized pattern of input and particular output [2]. In the approach, the diseased regions are analyzed by the input layer whereas the output layer identifies the infection effect on the affected section. Among the input and output layer, a hidden layer occurs. This layer provides concerning link amid the input and output images. This approach is implemented for achieving minimum error in the classification of infection of the pretentious area.

\section{RELATED WORK}

Abirami Devaraj, et.al (2019) stated that images processing techniques were used to detect plant infections. The major objective of this study was to develop a software system for automatic detection and classification of plant infections [3]. The image processing approach used various steps to identify plant diseases such as image acquisition, image preprocessing, image segmentation, feature extraction and image classification. In order to detect plant infections, the images of the leaves were utilized. Thus it was analyzed that image processing techniques were very beneficial in the detection and classification of infections in farming applications. In this study, an automatic detection system was developed with the help of higher technology such as image processing. This approach was developed to provide aid to the farmers for the early detection of plant infections. This approach also supplied useful data for its handling. In future, this study would be extended further for detecting large number of infections.

M S Arya, et.al (2018) presented a review of different image processing techniques to detect plant infections [5]. The major purpose of this study was to identify the infections. In order to detect infections, speed and accuracy were identified as two imperative aspects. Therefore, the expansion of this study would be focused on the development of modified algorithms for speedy and correct recognition of infectious leaves. In this study, a survey on various infection classification methods was performed. These classification techniques could be utilized to detect the infection of plant leaf. In this study, an algorithm for image segmentation method utilized for mechanical discovery and classification of plant leaf infection was discussed as well. Hence, correlated infections for these plants were utilized for detection purpose. The most excellent outcomes were achieved with minimum computational efforts. These results demonstrated the effectiveness of proposed algorithm in detection and classification of the plant infections

V Pooja, et.al (2017) proposed novel infection recognition and classification method using different machine learning methods and image processing techniques [6]. At first, the diseased part was identified and captured. After that, image preprocessing was applied on diseased part. Moreover, the segments were achieved and the region of interest was identified. In the next step, required features like color, shape, and contrast were extracted from the segmented image using the feature extraction technique. At last, the attained outcomes were provided by SVM Classification models for getting results. This classification model showed a very good performance in the classification of plant infection. The tested outcomes depicted that proposed approach showed significantly improved results as compared to earlier applied infection recognition approaches.

Santhosh Kumar S, et.la (2019) provided a comprehensive review of different plant infection detection algorithms [1]. It was analyzed that the recognition of infection was highly important for both plants and humans. Consideration of appropriate input was essential for having a significant effect of plant infection \& systems in the region of farming. In this study, various research problems were considered for developing a methodical technique to identify and distinguish the plant infections. These techniques would be very helpful for farmers and pathologists in future study. In this study, the significance of image processing techniques in agriculture field was described. In this work, different sorts of infections were also considered for additional study. Shivani K. Tichkule, et.al (2016) stated that timely and precise recognition of the plant infections was imperative for good and thriving development of crops [9]. It was analyzed that image processing techniques played an important role in the detection of plant diseases. With the help of image processing tools, the infections within different plants could be detected and classified precisely. In this work, K-means Clustering approach was used for the detection of diseased parts. On the other hand, Neural Networks were particularly utilized to obtain correctness in the detection and classification of infections. Therefore, these approaches could be utilized efficiently in Agrobot structure.

$\mathrm{R}$ Anand, et.al (2016) proposed a new technique to detect the infections on a plant leaf. In this work, one more approach was employed for watchful discovery of infections [10]. The main objective of proposed approach was to identify the infection of eggplant leaf with the help of image processing and artificial neural approaches. The infections on the eggplant were serious problems. Due to these infections, the production of eggplant crop was affected badly. In this work, main attention was given to the eggplant leaf instead of complete eggplant plant as around 85-95\% of infections occurred on the eggplant leaf. In this study, two techniques such as K-means clustering algorithm and Neural-network were used for detecting the infection on the leaf of the eggplant. The K-means clustering algorithm was used for segmentation purpose while classification was performed using Neural-network. It was 
analyzed that the proposed discovery scheme based on artificial neural networks proved extremely effectual in the detection of leaf infection.

\section{PROPOSED WORK}

This research work is based on the plant disease detection. The plant disease detection technique is based on the segmentation, feature extraction and classification. The methodology describes the complete process which is carried out in this research work.

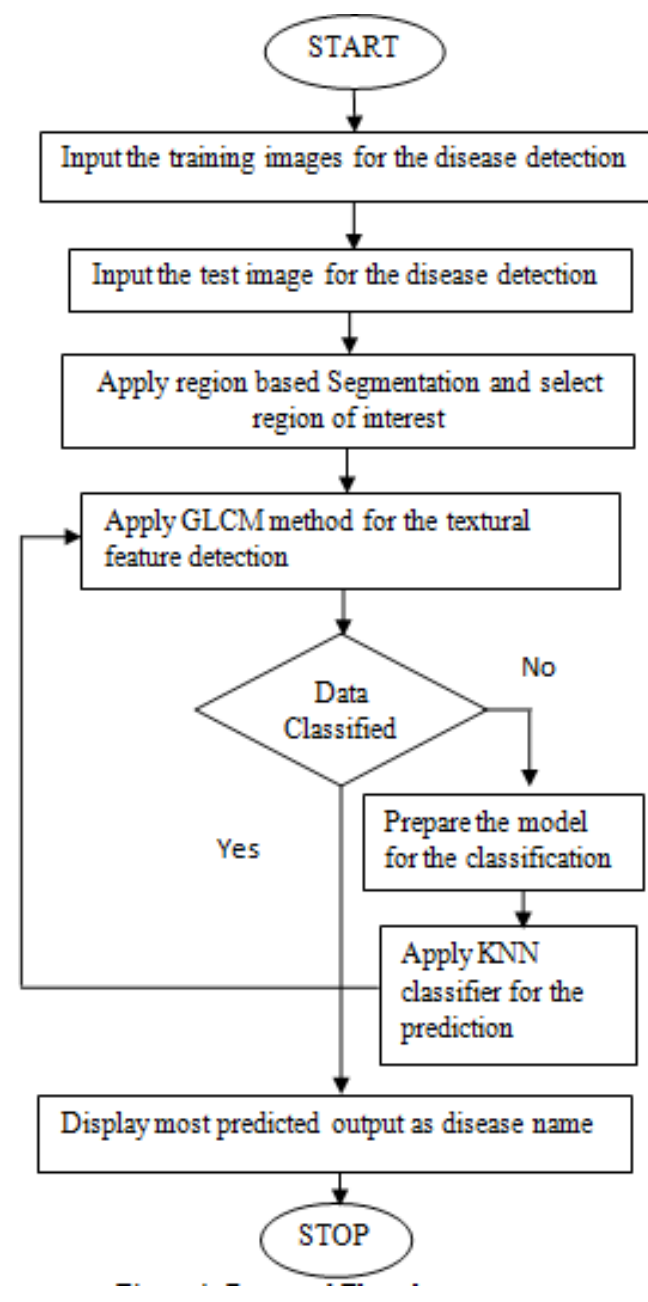

Figure 1: Proposed Flowchart

The stages depicted in the presented Flowchart are given below:

Stage 1: In the primary stage, the training pictures are used in the form of input for the identification of plant infection.

Stage 2: In the secondary stage, the characteristics of the training pictures are retrieved and amassed in the folder

Stage 3: The test picture is utilized as input and descriptions of the picture are retrieved by law texture algorithm in the third stage.

Stage 4: In the step four, the area based segmentation algorithm is implemented for the segmentation of test picture into definite domains.
Stage 5: In the last step, the KNN classifier is implemented in the final stage for the classification of the test picture into distinct classes in accordance with training set.

\section{RESUlTS AND DISCUSSION}

The proposed technique is implemented in MATLAB and the results are evaluated by making comparisons against proposed and existing techniques in terms of different parameters. The formula for the calculation of accuracy and execution time are described below:-

\section{A. Accuracy:}

For a particular program, the accurateness $i$ of the classification may be defined as the amount of patterns categorized accurately to the whole amount of patterns. The principle used is declared below

$$
\begin{gathered}
A=\frac{t}{i} n \\
n
\end{gathered}
$$

In this formula, $\mathrm{t}$ denotes the amount of pattern cases which are classified accurately and variable $\mathrm{n}$ depicts the amount of pattern suites.

\section{B. Execution Time:}

The execution time is described in the form of dissimilarity amid the end time when the execution of algorithm stops and the time at which the execution of algorithm commences.

Execution time $=$ End time of algorithm- start of the algorithm

The correctness of the projected method is improved in comparison with the accessible system as demonstrated by the figure 2. The accurateness of accessible method is 95 percent while the projected method gives a correctness rate of around 97 percent. A comparison on the basis of implemented time duration of the projected and accessible algorithm is carried out for the presentation scrutiny. It is discovered that projected algorithm utilizes less implementation time than accessible algorithm.

\section{Accuracy}

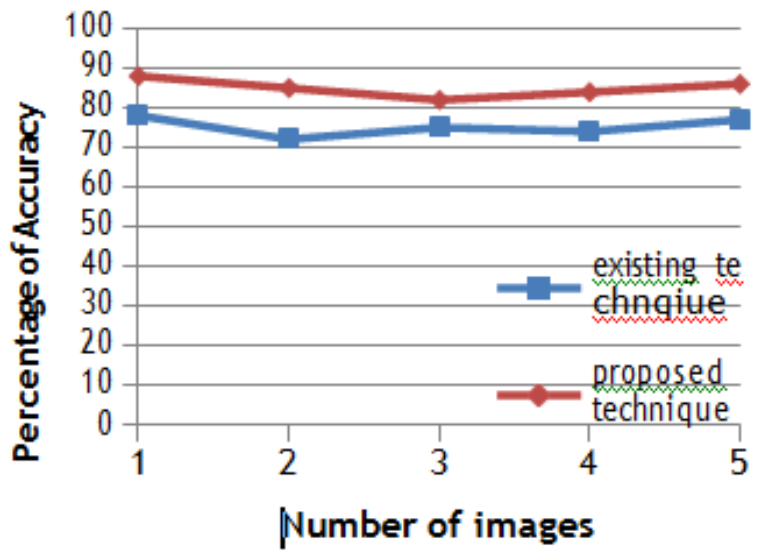

Fig 2: Accuracy Comparison. 


\section{Execution Time}

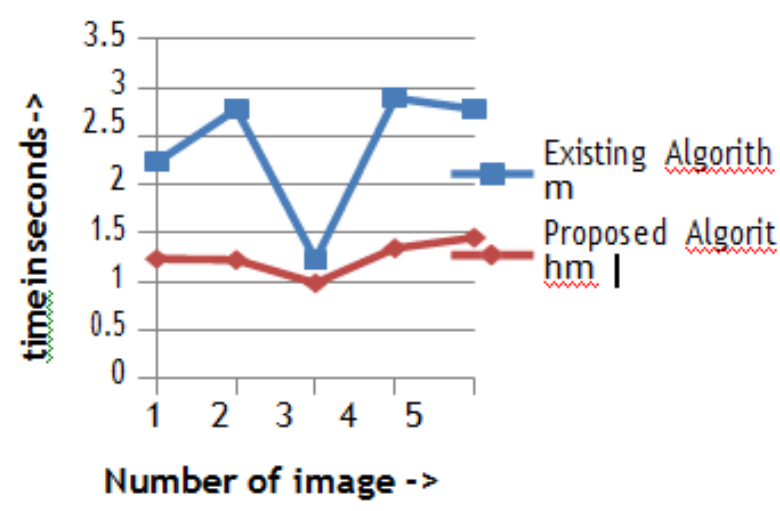

Fig 3: Execution Time Comparison

\section{CONCLUSION}

In this work, it is concluded that plant disease detection is approach which is applied to detect diseases from the leaves. The plant disease detection have various phases which are pre-processing, segmentation, feature extraction and classification. In the proposed method approach of $\mathrm{k}$-nearest neighbor is applied for the plant disease detection. The proposed model is implemented in MATLAB and results are analyzed in terms of accuracy and execution time. It is analyzed that accuracy of the proposed model is high and execution time is low as compared to existing technique

\section{REFERENCES}

1. Santhosh Kumar S, B. K. Raghavendra, "Diseases Detection of Various Plant Leaf Using Image Processing Techniques: A Review", 2019, 5th International Conference on Advanced Computing \& Communication Systems (ICACCS)

2. Kriti, Jitendra Virmani and Ravinder Agarwal, "Effect of despeckle filtering on classification of breast tumors using ultrasound images", Biocybernetics and Biomedical Engineering, Vol. 39, No. 2, pp. 536-560, 2019, Publisher: Elsevier

3. Abirami Devaraj, Karunya Rathan, Sarvepalli Jaahnavi, K Indira, "Identification of Plant Disease using Image Processing Technique", 2019, International Conference on Communication and Signal Processing (ICCSP)

4. Meenakshi Garg, Manisha Malhotra and Harpal Singh, "Statistical Feature Based Image Classification and Retrieval Using Trained Neural Classifiers", 2018, International Journal of Applied Engineering Research, Volume 13, Number 8, pp. 5766-5771

5. M S Arya, K Anjali, Divya Unni, "Detection of unhealthy plant leaves using image processing and genetic algorithm with Arduino", 2018, International Conference on Power, Signals, Control and Computation (EPSCICON)

6. V Pooja, Rahul Das, V Kanchana, "Identification of plant leaf diseases using image processing techniques", 2017, IEEE Technological Innovations in ICT for Agriculture and Rural Development (TIAR)

7. Sujatha R, Y Sravan Kumar and Garine Uma Akhil, "Leaf disease detection using image processing",2017, Journal of Chemical and Pharmaceutical Sciences

8. Sujeet Varshney, Tarun Dalal, "Plant Disease Prediction using Image Processing Techniques- A Review", 2016, International Journal of Computer Science and Mobile Computing

9. Shivani K. Tichkule, Dhanashri. H. Gawali, "Plant diseases detection using image processing techniques", 2016, Online International Conference on Green Engineering and Technologies (IC-GET)

10. R Anand, S Veni, J Aravinth, "An application of image processing techniques for detection of diseases on brinjal leaves using k-means clustering method", 2016, International Conference on Recent Trends in Information Technology (ICRTIT)

11. K. Sumithra, S. Buvana, R. Somasundaram, "A Survey on Various Types of Image Processing Technique", 2015, International Journal of Engineering Research \& Technology (IJERT)

12. Sachin D. Khirade, A.B. Patil, "Plant Disease Detection Using Image Processing", 2015, International Conference on Computing Communication Control and Automation

\section{AUTHORS PROFILE}

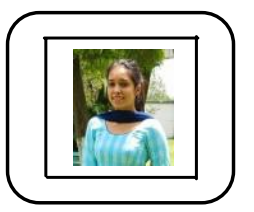

Simranjeet kaur. I have done my btech in computer science in 2017 from Rayat and Bahra Institute of Engineering and Technology kharar Mohali ,India. Currently I am pursuing my MTech from Chandigarh Group Of College ,Landran ,Mohali in the stream of computer science and my research work is in the area of Image Processing and Machine Learning.

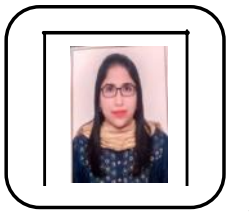

Geetanjali Babbar,Working as an Assistant Professor in Chandigarh Engineering College, Landran, Pursuing Ph.D in Computer Science, M.Tech in Computer Science, 16 national/international publications, Life time member of Indian Society for Technical Education. I have 15 years of teaching experience in CEC Landran. I have been awarded for best teacher in computer science department of CEC Landran. My research work is in the area of Machine Learning.

Dr. Gagandeep received his Bachelor's degree in Computer Science and Engineering from Punjab Technical University, Jalandhar, Punjab, India in 2002, M.E. degree in Computer Science and Engineering from PEC

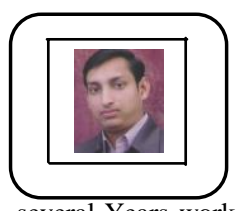
University of Technology, Chandigarh, India, in 2005 and $\mathrm{Ph} . \mathrm{D}$. degree in Computer Engineering from Panjabi University, Patiala, India in 2107. Presently he Works in Department of Computer s Science and Engineering, Chandigarh Engineering College (CEC),

Landran, Mohali, India as faculty member and has several Years work experience in the areas of teaching. He has published more than 40 research papers in leading journals, conference proceedings and books including Elsevier, IEEE, Taylor $n$ Francis and Springer and also holds 23 patents. He is also life member of several societies, received prestigious Academia award from CSI consecutively in 2011 \& 2012 and also received ISTE best teacher award in 2016. His current research interests include medical image processing, object detection, semantic retrieval, deep learning. 